\title{
Espacios de verificación en TikTok. Comunicación y formas narrativas para combatir la desinformación
}

\section{Fact-checking in TikTok. Communication and narrative forms to combat misinformation}

Pavel Sidorenko Bautista. Universidad Francisco de Vitoria. España.

pavel.sidorenko@ufv.es

$[\mathrm{CV}](1) \mathrm{G} \mathrm{R}^{\mathrm{T}} \mathrm{O}$

Nadia Alonso-López. Universitat Politècnica de València. España.

naallo1@har.upv.es

$[\mathrm{CV}]$ (1) $\mathrm{G} \mathrm{R}^{\mathrm{G}} \mathrm{O}$

Fábio Giacomelli. Universidad Beira Interior. Portugal.

fabio@ubi.pt

$\underline{\mathrm{CV}}] \mathrm{CR}^{\mathrm{i}}$

\begin{abstract}
Cómo citar este artículo / Referencia normalizada
Sidorenko-Bautista, P.; Alonso-López, N.; Giacomelli, F. (2021). Espacios de verificación en TikTok. Comunicación y formas narrativas para combatir la desinformación. Revista Latina de Comunicación Social, 79, 87-113. https://www.doi.org/10.4185/RLCS-2021-1522
\end{abstract}

\section{RESUMEN}

Introducción. Desde 2020, TikTok se ha convertido en red social tendencia a escala global. De ser inicialmente una plataforma para adolescentes, se ha convertido en un entorno digital donde impera la comunicación efímera, muy estimulante y visual. Hoy confluyen en ella una amplia diversidad de emisores, entre los cuales se encuentran medios y periodistas. Y, como ocurre en las redes sociales, en un contexto de hiperconectividad las informaciones falsas se viralizan con facilidad, contribuyendo así al desarrollo de la desinformación. Metodología. A partir de la combinación de técnicas de carácter cualitativo y cuantitativo, tales como estudio de casos, análisis de contenidos, entrevistas en profundidad y encuestas, se analiza la comunicación de los fact-checkers con perfil activo en TikTok, con el fin de determinar las claves para desmentir bulos y luchar contra la desinformación en una red social tan particular, donde aparentemente los usuarios acuden para divertirse y distraerse. Discusión y conclusiones. Queda en evidencia la pertinencia y la necesidad de que medios, periodistas y, específicamente, medios de verificación lleven a cabo su labor a través de nuevos escenarios como lo es TikTok, no solo para poder asegurar un incremento del alcance del mensaje de cara a nuevos segmentos de audiencia, sino como un proceso innovador para atacar el fenómeno de la desinformación, noticias falsas y especulaciones cada vez más descentralizado a través de los canales digitales. TikTok ha supuesto un reto narrativo a partir de la síntesis del mensaje y la creatividad visual.

PALABRAS CLAVE: Periodismo de verificación; Desinformación; Fact-checking; Noticias falsas; Nuevas narrativas; TikTok; Comunicación efímera. 


\begin{abstract}
Introduction. Since 2020, TikTok has turned into a global trending social network. It has become a digital environment where ephemeral, highly stimulating, and visual communication reigns from being initially a platform for teenagers. Today, it brings together a wide range of broadcasters, including the media and journalists. And, as it occurs in social networks, in a context of hyperconnectivity, false information is easily made viral, thus contributing to the development of disinformation. Methodology. From a combination of qualitative and quantitative techniques, such as case studies, content analysis, in-depth interviews, and surveys, the communication of factcheckers with an active profile on TikTok is analyzed in order to determine the keys to debunking hoaxes and combating disinformation on such a particular social network, where users apparently go to have fun and distract themselves. Discussion and conclusions. It is evident the relevance and needs for media, journalists, and the verification media to carry out their work through new scenarios such as TikTok. Not only to ensure a message reaching increase to new audience segments, but also as an innovative process to attack the phenomenon of disinformation, such as fake news and speculation that is increasingly decentralized through digital channels. TikTok has been a narrative challenge based on message synthesis and visual creativity.
\end{abstract}

KEYWORDS: Verification journalism; Misinformation; Fact-checking; Fake-news; New narratives; TikTok; Ephemeral communication.

\title{
CONTENIDO
}

1. Introducción. 2. Marco Teórico. 2.1. Noticias falsas, desinformación y bulos. 2.2 Combatir la desinformación: el fact-checking. 2.3 TikTok y el periodismo de verificación. 3. Metodología. 4. Resultados. 5. Discusión. 6. Conclusiones 7. Bibliografía

\section{Introducción}

Posverdad fue elegida palabra del año en 2016 por el diccionario Oxford. En la definición que realiza de este vocablo explica que denota circunstancias en las que los hechos que cuentan con una carga emocional e implican las propias creencias personales influyen más en la sociedad que los propios hechos objetivos. La posverdad es una sociedad que prefiere huir de la verdad (Tesich, 1992), donde la manipulación y las mentiras operan como mecanismos de protección.

Por su parte, Keyes (2004) se refiere a la Era de la Posverdad en un momento de expansión de Internet y las nuevas tecnologías de la Información y la Comunicación en que los usuarios pueden construir, reinterpretar y participar de sus propios relatos avanzando hacia una cultura online en la que resulta cada vez más difícil distinguir entre la realidad y la ficción (Coughland, 2017).

En un contexto de hiperconectividad y desarrollo tecnológico, las informaciones falsas pueden convertirse en virales y resulta altamente complicado desmentirlas debido al volumen de audiencia alcanzado y a la afinidad que pueden generar si se distribuyen a través de círculos que aportan credibilidad y peso emocional (Rodríguez-Fernández, 2021).

En este sentido, las redes sociales adquieren un papel relevante como vehículos de informaciones falsas. Pero ese potencial también puede emplearse para verificar estos contenidos desinformativos. De hecho, diversas agencias de verificación tienen presencia en redes sociales desde donde desmienten noticias falsas, bulos y otros contenidos desinformativos. 


\section{Marco Teórico}

Desinformación y posverdad se encuentran vinculadas al actual ecosistema mediático marcado por la hiperconectividad, el desarrollo tecnológico, los nuevos soportes y la expansión de las redes sociales.

Esto ha originado una variación en el flujo comunicativo y un aumento sin precedentes de las fuentes informativas, quedando los medios de comunicación desbancados en muchos casos por las redes sociales como fuente primaria de información (Coleman, Morrison y Anthony, 2012; Alonso, 2019), lo que contribuye a la difusión y viralización de contenidos falsos (Ceron, De Lima-Santos y Quiles, 2021; Jang y Kim, 2018). Por ello, y a pesar de no tratarse de un fenómeno nuevo (Salas, 2019; Vizoso y Vázquez, 2019), la desinformación ha adquirido una dimensión masiva en los últimos años, constituyendo un problema más importante de lo que lo fue en el pasado (Burkhardt, 2017; Guallar et al, 2020) al verse incrementadas las posibilidades de difusión de contenidos desinformativos y, por ende, las dificultades para determinar la certeza o no de una información (Blanco, García y Tejedor, 2019).

\subsection{Noticias falsas, desinformación y bulos}

En 2017, un año después de que posverdad fuese declarada palabra del año, tal consideración pasó al término fake news. La popularidad del término se incrementó a partir de los procesos electorales en Estados Unidos en 2016 que llevaron a la presidencia a Donald Trump y el proceso del Brexit que desembocó en la separación de Reino Unido de Europa (Allcott y Gentzkow, 2017; López-Borrull, 2020) siendo situados estos períodos por diversos estudios (Cabezuelo y Manfredi, 2019; MacKenzie y Bhatt, 2020, Pérez-Dasilva, Meso-Ayerdi y Mendiguren-Galdospin, 2020) como entre los más prolíficos en cuanto a producción de noticias falsas.

En este sentido, cabe tener en cuenta definiciones como la realizada por Amorós (2018) en la que se atribuye a las noticias falsas la finalidad de obtener un beneficio político o de otra índole. Por su parte, Salaverría et al (2020) se decantan por 'bulo' para referirse a estos contenidos falsos creados y difundidos deliberadamente con una intencionalidad que va desde la broma o la parodia hasta el engaño deliberado.

Por su carácter sensacionalista pero también por la implicación emocional que conllevan acorde a las creencias preexistentes de los usuarios (Albright, 2017; Mazaira, Rúas y Puentes, 2019; Preston et al., 2021), las noticias falsas obtienen una mayor credibilidad, se difunden con una gran rapidez y tienen un $70 \%$ más de posibilidades de ser replicadas "llegando más lejos, más rápido y más profunda y ampliamente que la verdad en todas las categorías de información." (RodríguezFernández, 2021: 39).

Respecto al término desinformación, refiere a un fenómeno más amplio y difícil de identificar (Salaverría, 2020; Del Fresno, 2019) que engloba todo contenido falso, inexacto o engañoso cuya difusión es intencionada con la finalidad de engañar, causar daño y obtener algún beneficio (European Comision, 2018; Lazer et al. 2018).

Por tanto, las noticias falsas se pueden entender como un género de la desinformación y un instrumento para la deslegitimación del periodismo (Egelhofer et al. 2020; McNair, 2017) en un momento de pérdida de confianza de los usuarios en la política y en los medios de comunicación tradicionales (Pérez-Curiel y Velasco-Molpeceres, 2020) en que la difusión de información falsa a través de las redes sociales constituye una amenaza potencial para la democracia y para la sociedad (Allcott, Gentzknow y Yu, 2019). 


\subsection{Combatir la desinformación: el fact-checking}

Ante la desconfianza sobre las informaciones de medios tradicionales y el crecimiento exponencial de la desinformación en la red, surge la necesidad de mecanismos de verificación que contribuyan a mejorar el acceso a la información de la ciudadanía (Palau-Sampio, 2018; Nyhan y Reiffler, 2015).

Por este motivo, en los últimos años se ha producido un importante crecimiento del fact-checking con iniciativas nacionales e internacionales (Alonso, 2019; Ufarte, Peralta y Murcia, 2018). Así, el fact-checking puede definirse como el mecanismo para analizar, contrastar y verificar informaciones que presenten una veracidad dudosa (García-Vivero y López, 2021) constituyendo la herramienta más eficaz para combatir la expansión de las informaciones falsas (Amorós, 2018).

Además, en agencias y medios de comunicación de referencia como el Washington Post, Le Monde, la BBC, Channel 4, la agencia EFE y Reuters, entre otros, han venido surgiendo medios independientes de verificación para que los usuarios puedan comprobar si una noticia es verdadera o falsa (Vázquez, Vizoso y López, 2019). Asimismo, iniciativas como The Trust Project suponen un compromiso de veracidad e imparcialidad en las informaciones al que se adhieren los diarios digitales. Y la herramienta informática TjTool, implementada por el diario Público.es (España), muestra a los usuarios la trazabilidad de los contenidos informativos que publica (Terol y Alonso, 2020).

Desde el ámbito institucional, destacan también estrategias como el Plan de Acción contra la Desinformación propuesto por la Unión Europea -en vigor desde 2019-, la creación de grupos de verificación para localizar contenidos desinformativos en redes sociales, la creación del Observatorio Europeo de Medios Digitales (EDMO) y la Red Internacional de Verificación de Datos (IFCN) del Instituto Poynter en Estados Unidos.

Asimismo, el aumento de informaciones falsas que se produjo a causa de la pandemia provocada por la Covid-19 (Sánchez y Magallón, 2020) instó a redes sociales como Facebook, Twitter y TikTok a implementar acciones coordinadas para desmentir los contenidos falsos en sus propias plataformas.

\subsection{TikTok y el periodismo de verificación}

La red social TikTok se caracteriza por una lógica de contenidos basados en el entretenimiento con grandes cantidades de estímulo visual, mucho dinamismo, exigencia creativa y rapidez de producción y consumo de publicaciones mayormente determinadas por playbacks, historias divertidas y contenidos "relajantes a la mente" (Li, Xiaohui y Zhegwu, 2019; Shuai, Yuzhen y Yifang, 2019; Yu-Liang, Chun-Chin y Shu-Ming, 2019). Es la consecuencia de un contexto comunicacional donde se abole la unidireccionalidad del mensaje, así como los roles preestablecidos desde la pasividad de emisores y receptores (Torres y Villena, 2020).

En otras palabras, se trata de un entorno digital que invita constantemente a la acción e implicación de los usuarios, cuya comunidad está compuesta en gran medida por la 'Generación Z' (Rapkin, 2017; Shuai et al., 2019) aunque el inicio de la pandemia por Covid-19 ocasionó que un importante número de Millennials y usuarios de la 'Generación X' se sumaran a esta vía no solo para consumir contenidos, sino para producirlos en similar proporción. 
Su app móvil ha sido la más descargada del 2020 (Ditrendia, 2020) y su rápida popularidad motivó a emisores de muy diversa naturaleza, entre ellos medios y periodistas (Sidorenko et al., 2020; Vázquez et al., 2020), a intentar innovar adaptándose rápidamente al particular código del mensaje.

De hecho, ha sido un canal digital muy importante en la distribución de información relacionada con la pandemia por Covid-19 (Basch, Hyllyer y Jaimie, 2020) no solo porque la Organización Mundial de la Salud, Cruz Roja y otros actores oficiales empezaron a actuar de manera muy temprana a través de ella, sino también por el uso de determinados hashtags empleados por muchos usuarios particulares. Esto ha permitido a los especialistas una mayor aproximación a los hábitos de los usuarios relacionados con la pandemia, con las impresiones y riesgos desinformativos que este tipo de entornos digitales conllevan.

Pese a la eventual impresión sobre la superficialidad que pueden reportar muchas publicaciones que circulan con éxito y popularidad a través de esta vía, Van den Bremer y Siebelink (2020) aprecian que los usuarios de TikTok tienen una visión y participación más crítica sobre los contenidos solo cuando se interesan verdaderamente en el tema. Una de las prácticas más habituales al respecto la constituye la revisión del cajón de comentarios para contrastar el contenido.

En TikTok la mayoría de los usuarios se preocupan mucho por su imagen y marca personal, por lo que en muchas ocasiones piensan lo que van a publicar (Vogel y Rose, 2016; Omar y Dequan, 2020). Esto puede -aunque no sea necesariamente así- incidir en la veracidad de los relatos.

Sin embargo, ante el ascenso sostenido de la actividad en esta plataforma a partir de 2020 (Ditrendia, 2020), los responsables de la red social han intentado controlar el flujo de vídeos especulativos y tendenciosos (Ballesteros, 2020; Pérez, 2021). De la misma manera que viene ocurriendo en otras plataformas digitales, en TikTok se ha empezado a reportar un flujo importante de contenidos que fomentan la desinformación de manera accidental, pero, sobre todo, también de forma intencionada. Desde 2021, denotando la importancia de la comunidad de usuarios, la red social ha tomado la determinación de eliminar inmediatamente las publicaciones que sean reportadas como 'bulos' y 'noticias falsas' (Pérez, 2021).

\section{Metodología}

Los estudios de Sidorenko, Herranz y Cantero (2020) y Vázquez, Negreira y López (2020) han dejado en evidencia el trabajo de diversos medios de comunicación a nivel global a través de TikTok, pese a las particularidades narrativas que dicha red pueda suponer.

Ante esta necesidad de incrementar el alcance del mensaje y poder llegar a nuevos segmentos de audiencia, así como para luchar contra la desinformación, el periodismo ha empezado a tener presencia en TikTok mediante el denominado periodismo de verificación, cada vez más activo frente al alto flujo de desinformación, bulos, especulaciones y noticias falsas que circulan a través de diversas vías digitales.

Esta investigación propone evidenciar cómo comunican estos fact-checkers en una red social donde impera el contenido efímero y veloz con alto grado de estímulos visuales. Para ello, se plantean las siguientes preguntas de investigación:

RQ1: ¿Qué temas abordan los fact-checkers en las publicaciones de TikTok?

RQ2: ¿Cómo son los contenidos que construyen los fact-checkers en esta red social?

RQ3: ¿Cómo reacciona la comunidad de usuarios a estos contenidos? 
RQ4: ¿TikTok es una moda para los fact-checkers o, por el contrario, es una vía más para atacar la desinformación?

A partir de estas preguntas, se establecen siguientes hipótesis:

H1. El trabajo de los fact-checkers en TikTok es incipiente y limitado en cuanto a actores.

H2. La particularidad del código del mensaje en TikTok limita el trabajo de los fact-checkers en la red social.

H3. La pandemia por Covid-19 y la inestabilidad política en diversos países ha aumentado la labor de fact-checkers en TikTok en torno a estos dos temas.

H4. El trabajo de fact-checkers a través de TikTok se ha centrado en verificar y desmentir bulos y noticias falsas.

H5. Pese a la importancia de la comunidad de usuarios en la dinámica de TikTok, los factcheckers interactúan poco con su audiencia.

Para dar respuesta a las preguntas e hipótesis planteadas y dado que TikTok es una red social en auge y relativamente inexplorada desde una perspectiva académica, se propone una metodología de estudio adaptada a la particularidad de la red social que consiste en la combinación de técnicas cuantitativas y cualitativas (Gaitán y Piñuel, 1998: 286; Soler y Enríquez, 2012: 887) a partir del rastreo de medios y fact-checkers con perfil activo en TikTok. La selección se ha realizado en base al listado presentado por la Fundación Gabo (2020, Abril, 2) sobre medios que han venido contrastando y verificando información frente a la infodemia de la Covid-19 y a la relación de medios que integran la International Fact-Checkers Network (IFCN, n.d). Se excluyen de la muestra los medios en proceso de revisión de afiliación por parte de la IFCN.

Tras una revisión entre más de cien nombres hasta el 28 de febrero de 2021, los fact-checkers con actividad en TikTok propuestos para estudio son:

- Bolivia Verifica @ boliviaverifica (Bolivia)

- Agencia Lupa @agencialupa (Brasil)

- Mala Espina @ malaespinacheck (Chile)

- Maldito Bulo@malditobulo (España)

- Newtral @newtraltiktok (España)

- Politifact @ politifact (Estados Unidos)

- France Info @franceinfo (Francia)

- Newscheckerin @newscheckerin (India)

- The Quint @ thequint (India)

- Kompas.com @kompascom (Indonesia)

- Tempo.co@tempodotco (Indonesia)

- Africa Check @africacheck (Sudáfrica)

- Factcheckvn @factcheckvn (Vietnam)

A partir de la información obtenida y con el fin de conocer la actividad y alcance de cada perfil, se ha creado una tabla de análisis que permita visualizar variables como: cantidad de seguidores, total de 'me gusta' y total de contenidos publicados desde el inicio de actividades en TikTok hasta la fecha límite de observación propuesta.

Se ha procedido a efectuar un análisis de los contenidos de cada medio y fact-checkers con la finalidad de determinar si su propuesta se adapta a la "narrativa TikTok" y constituye una labor diferenciada con respecto a los contenidos que desarrollan a través de otras plataformas digitales. Asimismo, se ha intentado delimitar qué tipo de temas se abordan con más frecuencia a través de esta plataforma particular. 
Los contenidos encontrados se han categorizado como:

- Contenido propio de TikTok - CP

- Réplica a comentarios en TikTok u otras redes sociales - RC

- Contenido proveniente de otra plataforma - COP

Se entiende por 'Contenido propio de TikTok' (CP) aquel que ha sido desarrollado en una narrativa vertical, según establece la red social. Por su parte, las 'Réplicas a comentarios de TikTok u otras redes sociales' (RC) son aquellas publicaciones que responden en vídeo a un comentario de un usuario en otra anterior (caso TikTok) o que hagan alusión expresa a una consulta realizada al medio a través de vías alternativas como Twitter, Facebook, Instagram o YouTube, por ejemplo. Por último, el 'Contenido proveniente de otra plataforma' (COP) es aquel que no respeta la narrativa de TikTok y ha sido publicado a través de esta vía en formato horizontal, cuadrado u otra dimensión que corresponda a otra plataforma digital.

Esta última categoría delimita si el medio hace un esfuerzo por realizar una comunicación diferenciada y adaptada a TikTok o simplemente desarrolla un contenido base y lo publica en similares condiciones a través de los canales digitales deseados.

Asimismo, para conocer sobre qué temas hacen mayor énfasis los fact-checkers estudiados, se han establecido las siguientes categorías:

- Temas políticos - A

- Temas sanitarios - B

- Temas de interés social - C

- Deportes - D

- Desinformación y Fake News en general - E

- Información propia - F

- Otros temas - G

- Recopilación de varios temas - H

Por otra parte, se han realizado entrevistas estructuradas a los responsables de algunos de estos perfiles con el fin de conocer sus motivaciones y estrategias para afrontar la lucha contra la desinformación en una plataforma con un código de mensaje tan particular como lo es TikTok. Así, se logró obtener las consideraciones de Maldita.es (España), Newtral (España), Mala Espina (Chile), Agencia Lupa (Brasil) y de Cotejo (Venezuela), este último como fact-checker ajeno a la red social pero inmerso en una realidad nacional con un alto flujo de desinformación. Este testimonio permite comprender la eventual importancia de TikTok aunque de momento no tengan participación activa.

Asimismo, de manera complementaria y con el fin de contrastar y/o complementar los datos obtenidos a través del estudio de los perfiles seleccionados, así como la información recibida a partir de las entrevistas, se realizó con intención meramente referencial y sin que los datos obtenidos resulten determinantes, una encuesta que contó con la participación de 400 personas en España, Brasil y Portugal. Las encuestas fueron realizadas durante el primer trimestre de 2021 a personas de diferentes perfiles de audiencia en posesión de un dispositivo móvil de comunicación. El primer paso fue caracterizar, de forma puramente referencial, al público que participó en la encuesta realizando una separación por edades (Gráfico 1) para delimitar el nivel de conocimiento de los usuarios acerca del término fact-checking y sus implicaciones, el alcance que tienen los fact-checkers a través de la plataforma TikTok y conocer los intereses de los usuarios en dicha red social para así concretar hasta qué punto estas labores de verificación son pertinentes a través de dicha plataforma. 
RLCS, Revista Latina de Comunicación Social, 79, 87-113

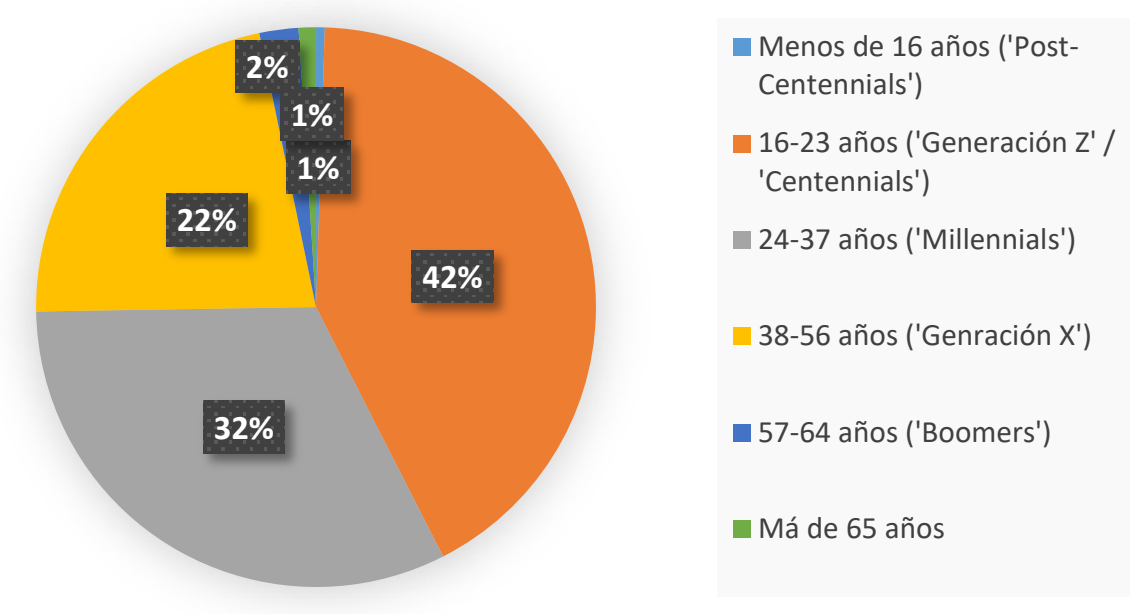

Gráfico 1: Distribución etaria de los encuestados para la investigación

Fuente: Elaboración propia

Como última nota aclaratoria, las revisiones de contenido de los medios indios, indonesios y vietnamita se han realizado con la asistencia de traductores en línea en tiempo real.

\section{Resultados}

A excepción de algunos casos, en primera instancia es posible apreciar que la mayoría de factcheckers comenzó su trabajo a través de TikTok en el contexto del primer año de la pandemia por Covid-19 (Tabla 1), es decir, en el momento en que la red social se convirtió en tendencia global, confirmándose así la primera hipótesis de la investigación. 
RLCS, Revista Latina de Comunicación Social, 79, 87-113

[Investigación] DOI: 10.4185/RLCS-2021-1522| ISSN 1138-5820| Año 2021

Tabla 1: Métricas de los perfiles de fact-checkers en TikTok desde la primera publicación hasta el 28 de febrero 2021

\begin{tabular}{|c|c|c|c|}
\hline $\begin{array}{c}\text { Id. del Fact-checker y fecha de inicio en } \\
\text { TikTok }\end{array}$ & $\begin{array}{c}\mathrm{N}^{\circ} \text { de } \\
\text { seguidores }\end{array}$ & $\begin{array}{l}\text { Total de 'me } \\
\text { gusta' }\end{array}$ & $\begin{array}{l}\mathrm{N}^{\circ} \text { de contenidos hasta el } 30 \mathrm{de} \\
\text { enero } 2021\end{array}$ \\
\hline $\begin{array}{c}\text { Bolivia Verifica } \\
\text { @ boliviaverifica } \\
10 / 02 / 2021\end{array}$ & 127 & 269 & 7 \\
\hline $\begin{array}{c}\text { Agencia Lupa } \\
\text { @agencialupa } \\
07 / 09 / 2020\end{array}$ & 11.600 & 72.411 & 106 \\
\hline $\begin{array}{c}\text { Mala Espina } \\
\text { @ malaespinacheck } \\
21 / 07 / 2020\end{array}$ & 19 & 276 & 11 \\
\hline $\begin{array}{c}\text { Maldito Bulo } \\
@ \text { malditobulo } \\
\text { 03/07/2019 }\end{array}$ & 7394 & 69.699 & 115 \\
\hline $\begin{array}{c}\text { Newtral } \\
\text { @ newtraltiktok } \\
19 / 10 / 2020\end{array}$ & 596 & 950 & 55 \\
\hline $\begin{array}{c}\text { Politifact } \\
\text { @ politifact } \\
30 / 08 / 2020\end{array}$ & 823 & 4558 & 15 \\
\hline $\begin{array}{l}\text { France Info } \\
@ \text { franceinfo } \\
11 / 11 / 2020\end{array}$ & 58.600 & 855.603 & 75 \\
\hline $\begin{array}{c}\text { Newscheckerin } \\
\text { @ newscheckerin } \\
18 / 06 / 2020\end{array}$ & 149 & 140 & 10 \\
\hline $\begin{array}{l}\text { The Quint } \\
\text { @ thequint } \\
16 / 10 / 2019\end{array}$ & 20.700 & 20.700 & 220 \\
\hline $\begin{array}{c}\text { Kompas.com } \\
\text { @ kompascom } \\
26 / 08 / 2020\end{array}$ & 141.700 & 136.317 & 293 \\
\hline $\begin{array}{l}\text { Tempo.co } \\
\text { @ tempodotco } \\
12 / 02 / 2020\end{array}$ & 315.200 & 2.766 .891 & 398 \\
\hline $\begin{array}{c}\text { Africa Check } \\
\text { @ africacheck } \\
30 / 03 / 2020\end{array}$ & 697 & 1834 & 16 \\
\hline $\begin{array}{l}\text { Factcheckvn } \\
@ \text { factcheckvn } \\
28 / 03 / 2020\end{array}$ & 85.700 & 336.912 & 79 \\
\hline
\end{tabular}

Fuente: Elaboración propia

El engagement obtenido a través del botón 'me gusta' es una variable irregular entre los diferentes fact-checkers consultados, como lo ha sido también la cantidad de publicaciones. De hecho, algunos llevan cierto tiempo sin volver a publicar en TikTok (hasta la fecha de la observación) mientras que en otras plataformas de tipo social o webs tienen una actividad actualizada:

- Politifact no publica desde el 17 dic 2020.

- Mala Espina no publica desde el 15 octubre 2020.

- Newscheckerin no publica desde el 19 junio 2020.

- The Quint no publica desde el 28 junio 2020.

- Africa Check no publica desde el 25 noviembre 2020. 
En cuanto al tema narrativo, fue posible identificar la actividad de los fact-checkers según los siguientes indicadores:

Tabla 2: Tipos de contenidos y temática abordadas por los fact-checkers en TikTok desde la primera publicación hasta el 28 de febrero 2021

\begin{tabular}{|c|c|c|c|c|c|c|c|c|c|c|c|}
\hline $\begin{array}{c}\text { Fact- } \\
\text { checker }\end{array}$ & $\begin{array}{c}\text { Tipo de } \\
\text { contenido }\end{array}$ & $\mathbf{A}$ & B & C & D & $\mathbf{E}$ & $\mathbf{F}$ & $\mathbf{G}$ & $\mathbf{H}$ & $\begin{array}{l}\text { Vídeos de } \\
\text { verificación }\end{array}$ & $\begin{array}{c}\text { Vídeos } \\
\text { informativo } \\
\text { s/ } \\
\text { educativos }\end{array}$ \\
\hline \multirow{3}{*}{$\begin{array}{c}\text { Bolivia } \\
\text { Verifica } \\
\text { @ boliviaver } \\
\text { ifica }\end{array}$} & $\mathrm{CP}$ & 6 & - & - & - & - & 1 & - & - & - & 7 \\
\hline & $\mathrm{RC}$ & - & - & - & - & - & - & - & - & - & - \\
\hline & COP & - & - & - & - & - & - & - & - & - & - \\
\hline \multirow{3}{*}{$\begin{array}{c}\text { Agencia } \\
\text { Lupa } \\
\text { @agencialu } \\
\text { pa }\end{array}$} & CP & 20 & 23 & 1 & - & 6 & 28 & 6 & 20 & 60 & 44 \\
\hline & $\mathrm{RC}$ & - & 2 & - & - & - & - & - & - & 2 & - \\
\hline & COP & - & - & - & - & - & - & - & - & - & - \\
\hline \multirow{3}{*}{$\begin{array}{c}\text { Mala } \\
\text { Espina } \\
\text { @ malaespin } \\
\text { acheck }\end{array}$} & $\mathrm{CP}$ & 7 & 3 & - & - & - & - & - & - & 3 & 7 \\
\hline & $\mathrm{RC}$ & - & - & - & - & - & - & - & - & - & - \\
\hline & COP & - & - & 1 & - & - & - & - & - & - & 1 \\
\hline \multirow{3}{*}{$\begin{array}{c}\text { Maldito } \\
\text { Bulo } \\
@ \underset{\text { malditobu }}{\text { lo }}\end{array}$} & $\mathrm{CP}$ & 3 & 31 & 12 & 2 & 20 & 7 & 20 & 4 & 41 & 58 \\
\hline & $\mathrm{RC}$ & - & 9 & 1 & - & 1 & - & 2 & - & 11 & 2 \\
\hline & COP & 1 & - & 1 & - & 1 & - & - & - & - & 3 \\
\hline \multirow{3}{*}{$\begin{array}{c}\text { Newtral } \\
\text { @ newtraltik } \\
\text { tok }\end{array}$} & CP & 5 & 5 & 5 & - & 3 & 2 & 9 & 3 & 11 & 21 \\
\hline & $\mathrm{RC}$ & - & - & - & - & - & - & - & - & - & - \\
\hline & COP & 3 & 8 & 6 & - & - & 2 & 6 & - & - & 25 \\
\hline \multirow{3}{*}{$\begin{array}{l}\text { Politifact } \\
\text { @ politifact }\end{array}$} & $\mathrm{CP}$ & 7 & 2 & - & - & - & 3 & 1 & 1 & 10 & 4 \\
\hline & $\mathrm{RC}$ & - & - & - & - & - & - & - & - & - & - \\
\hline & COP & 1 & - & - & - & - & - & - & - & 1 & - \\
\hline \multirow{3}{*}{$\begin{array}{l}\text { France Info } \\
\text { @franceinfo }\end{array}$} & $\mathrm{CP}$ & 4 & 13 & 31 & - & - & 1 & 23 & 1 & 17 & 56 \\
\hline & $\mathrm{RC}$ & - & - & - & - & - & - & - & - & - & - \\
\hline & COP & - & - & 1 & - & - & - & 1 & - & - & 2 \\
\hline \multirow{3}{*}{$\begin{array}{c}\text { Newschecke } \\
\text { rin } \\
\text { @ newschec } \\
\text { kerin }\end{array}$} & $\mathrm{CP}$ & - & 8 & - & - & 1 & 1 & - & - & - & 10 \\
\hline & $\mathrm{RC}$ & - & - & - & - & - & - & - & - & - & - \\
\hline & COP & - & - & - & - & - & - & - & - & - & - \\
\hline \multirow{3}{*}{$\begin{array}{l}\text { The Quint } \\
\text { @ thequint }\end{array}$} & $\mathrm{CP}$ & 2 & 13 & 25 & - & - & 2 & 19 & 1 & 20 & 42 \\
\hline & $\mathrm{RC}$ & - & - & - & - & - & - & - & - & - & - \\
\hline & COP & 6 & 13 & 62 & 1 & - & 2 & 73 & 1 & - & 158 \\
\hline
\end{tabular}


RLCS, Revista Latina de Comunicación Social, 79, 87-113

[Investigación] DOI: 10.4185/RLCS-2021-1522| ISSN 1138-5820| Año 2021

\begin{tabular}{|c|c|c|c|c|c|c|c|c|c|c|c|}
\hline \multirow{3}{*}{$\begin{array}{c}\text { Kompas.co } \\
\text { m } \\
\text { @ kompasco } \\
\text { m }\end{array}$} & $\mathrm{CP}$ & 50 & 24 & 72 & 5 & 1 & 2 & 135 & 1 & 10 & 280 \\
\hline & $\mathrm{RC}$ & - & - & - & - & - & - & - & - & - & - \\
\hline & COP & 2 & - & - & - & - & - & 1 & - & - & 3 \\
\hline \multirow{3}{*}{$\begin{array}{c}\text { Tempo.co } \\
\text { @tempodot } \\
\text { co }\end{array}$} & $\mathrm{CP}$ & 136 & 42 & 91 & 1 & - & 16 & 95 & 18 & 32 & 367 \\
\hline & $\mathrm{RC}$ & - & - & - & - & - & - & - & - & - & - \\
\hline & $\mathrm{COP}$ & 3 & 6 & 13 & - & - & - & 9 & - & - & 31 \\
\hline \multirow{3}{*}{$\begin{array}{c}\text { Africa } \\
\text { Check } \\
\text { @africachec } \\
\text { k }\end{array}$} & $\mathrm{CP}$ & - & 6 & - & - & 8 & 1 & - & - & 4 & 11 \\
\hline & $\mathrm{RC}$ & - & - & - & - & - & - & - & - & - & - \\
\hline & $\mathrm{COP}$ & - & 1 & - & - & - & - & - & - & 1 & - \\
\hline \multirow{3}{*}{$\begin{array}{c}\text { Factcheckvn } \\
@ \text { factcheck } \\
\text { vn }\end{array}$} & $\mathrm{CP}$ & 10 & 29 & 14 & - & 1 & 1 & 22 & 1 & 76 & 2 \\
\hline & $\mathrm{RC}$ & - & - & - & - & - & - & - & - & - & - \\
\hline & $\mathrm{COP}$ & - & - & 1 & - & - & - & - & - & 1 & - \\
\hline \multicolumn{2}{|c|}{ TOTAL } & 266 & 238 & 337 & 9 & 42 & 69 & 422 & 51 & 300 & 1.134 \\
\hline
\end{tabular}

(CP) Contenido propio de TikTok | (RC) Réplica a comentarios en TikTok u otras redes sociales | (COP) Contenido proveniente de otra plataforma

A) Temas políticos |B) Temas sanitarios |C) Temas de interés social |D) Deportes |E) Desinformación y Fake News |F) Información propia |G) Otros temas |H) Recopilación de varios temas

Fuente: elaboración propia

Según los datos de la Tabla 2 y el Grafico 2, y respondiendo a la RQ1, el mayor flujo de contenidos abordados por los fact-checkers estudiados corresponde a la categoría "G" que alude a "Otros temas", entre los cuales hay informaciones de índole económico, climático y tecnológico, entre otros. Seguidamente, los temas de interés social ("C"), políticos ("A") y sanitarios ("B"), en ese orden, constituyen los otros temas con mayor volumen de contenidos dedicados.

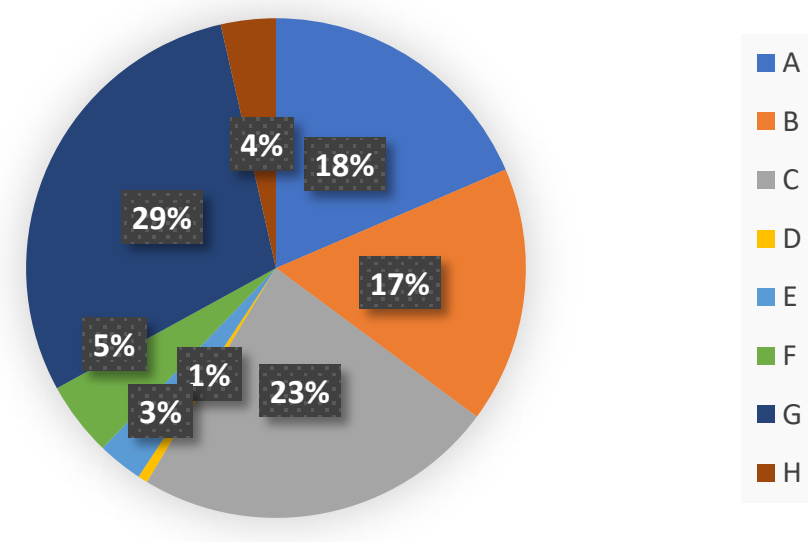

Gráfico 2: Total de temáticas abordadas en TikTok por los fact-checkers desde su primera publicación hasta el 28 de febrero 2021

Fuente: elaboración propia 
En relación a la H3, según datos de la Tabla 2 y Grafico 3, 12 de los 13 fact-checkers estudiados elaboraron contenidos en TikTok centrados en el tema salud, siendo la coyuntura de la Covid-19 una las principales variables y consecuencias de la producción de publicaciones centradas en este ámbito. Destacan así los fact-checkers españoles Maldito Bulo y Newtral, así como la brasileña Agencia Lupa y la vietnamita Factcheckvn, que han concentrado su atención en mayor medida en el tema sanitario, fuertemente influenciado por dicha pandemia.

Por su parte, Newscheckerin, de India, solo ha producido contenido en TikTok centrado en el tema de la Covid-19, mientras que The Quint, también de India, pese a tener una importante producción de contenidos influenciados por dicha coyuntura sanitaria, ha centrado sus esfuerzos en el tema social, determinado también de manera importante por la pandemia.

Asimismo, 11 de los 13 perfiles publicaron contenidos sobre temas políticos, tanto de interés nacional como internacional.

Los fact-checkers indonesios concentraron principalmente sus esfuerzos en temas políticos y sociales, tales como denuncias por casos de corrupción, presuntos abusos de poder en administraciones locales, manifestaciones, desplazados, migrantes y exclusión social, entre otros.

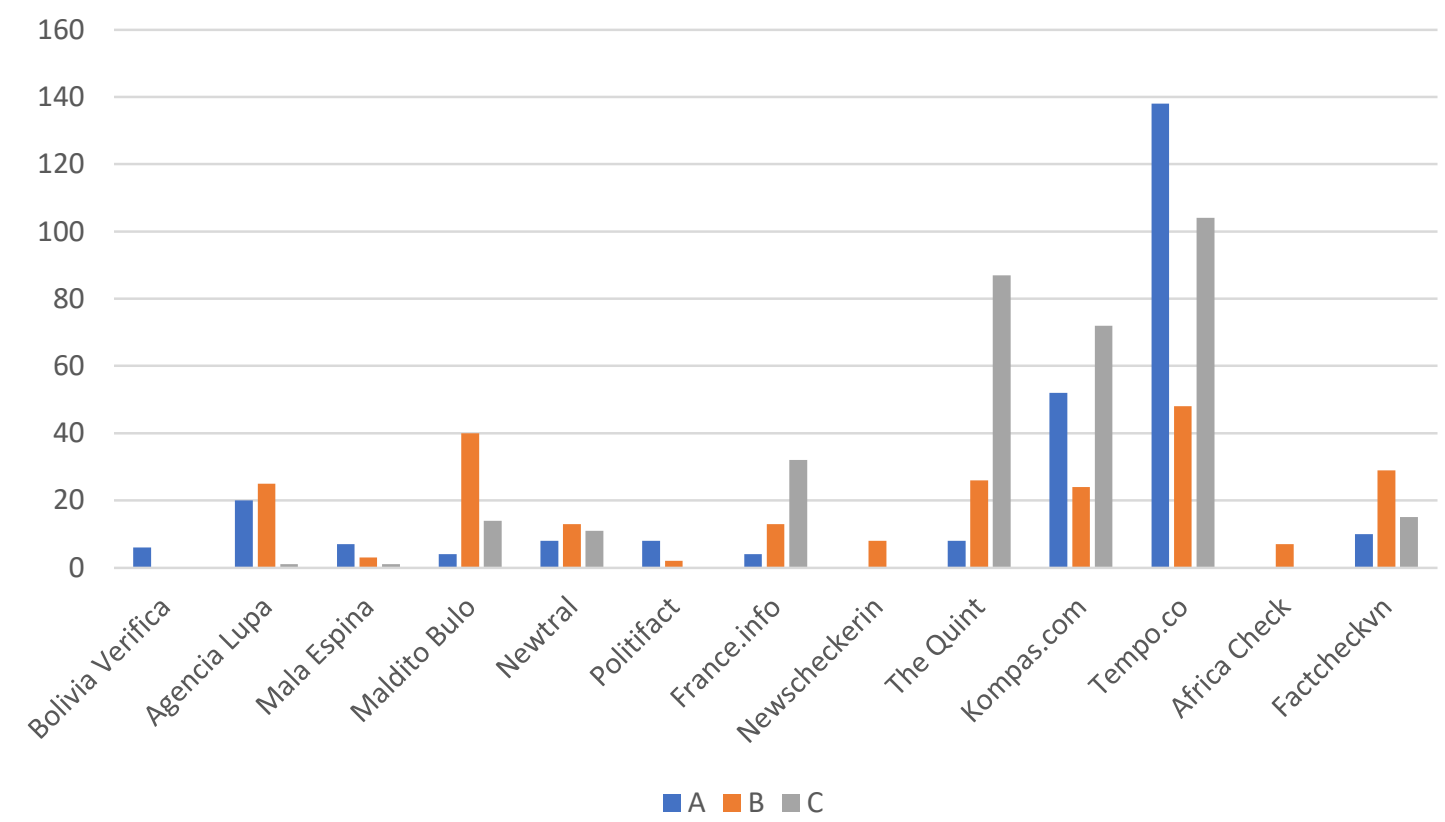

Gráfico 3: Producción de contenidos sobre las tres principales temáticas: Política, Salud y Social por parte de los fact-checkers en TikTok desde su inicio de actividades en la red social hasta el 28 de febrero 2021

Fuente: elaboración propia

La mayoría de los fact-checkers estudiados en TikTok realizan contenidos donde muestran sus rutinas de trabajo y la labor que adelantan en torno a la desinformación y las noticias falsas. Muchos de estos vídeos son en clave de humor, perfectamente adaptados a la estética y código del mensaje de la red social. 
No obstante, casos como Maldito Bulo (22 contenidos), Africa Check (8 contenidos), Agencia Lupa (6 contenidos), Newtral (3 contenidos), Newscheckerin (1 contenido), Kompas.com (1 contenido) y Factcheckvn (1 contenido) también han invertido tiempo y esfuerzos -unos mucho más que otros como se aprecia en la Tabla 2 y Gráfico 4- en producir contenidos que buscan educar sobre el fenómeno de la desinformación, noticias falsas, bulos y especulaciones que abundan sobre todo a través de vías digitales.

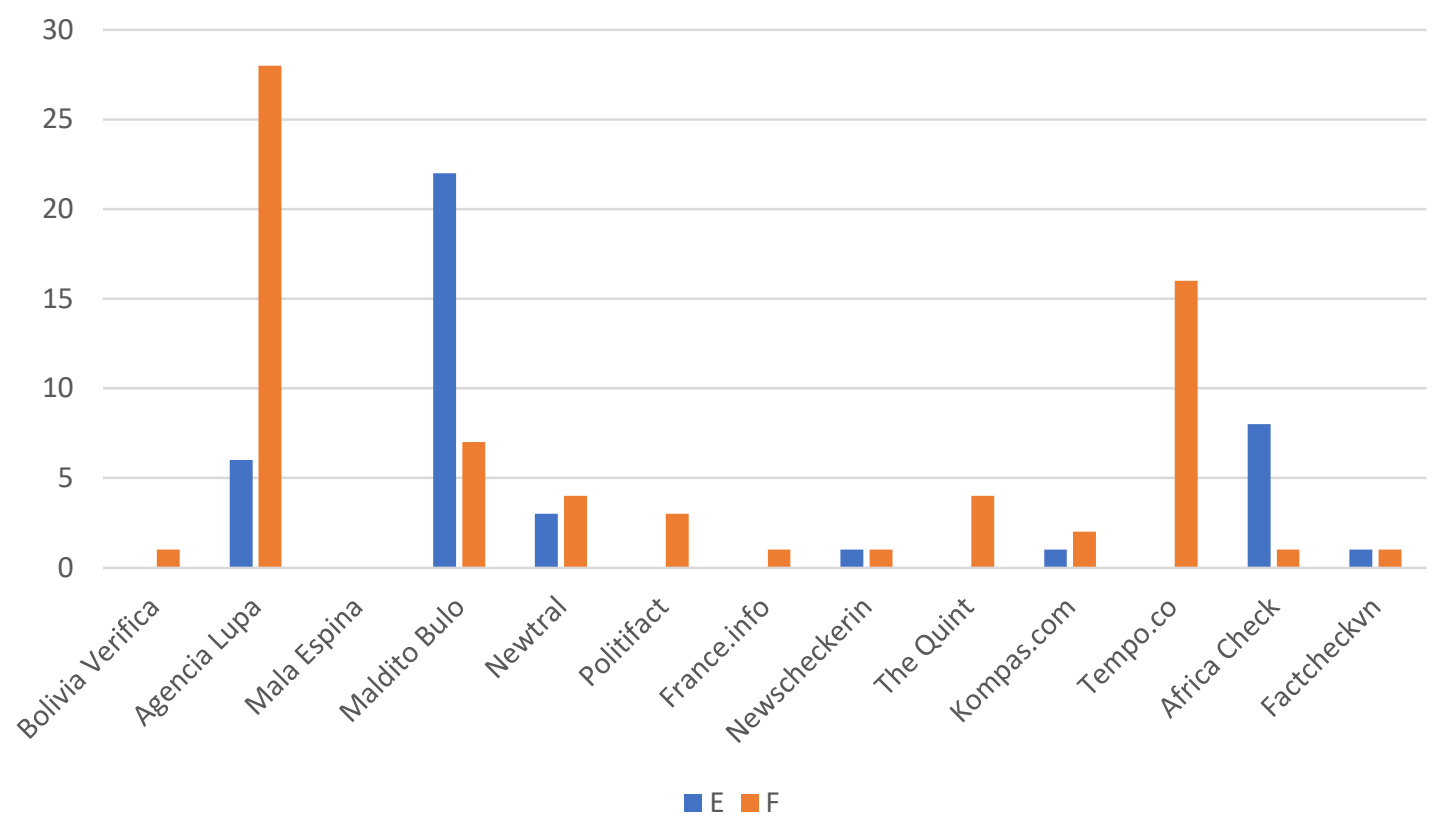

Gráfico 4: Contenidos educativos y orientativos sobre desinformación y fake news, y contenidos promocionales de la labor de los fact-checkers desde el inicio de actividades en TikTok hasta el 28 de febrero 2021

Fuente: elaboración propia

En atención a lo indicado en el Gráfico 5, a excepción de Agencia Lupa (Brasil) y Factcheckvn (Vietnam), los fact-checkers publican en TikTok más con intención informativa y orientativa que para la propia verificación de bulos, desinformación y noticias falsas, con lo que no se cumple la H4. 
RLCS, Revista Latina de Comunicación Social, 79, 87-113

[Investigación] DOI: 10.4185/RLCS-2021-1522| ISSN 1138-5820| Año 2021

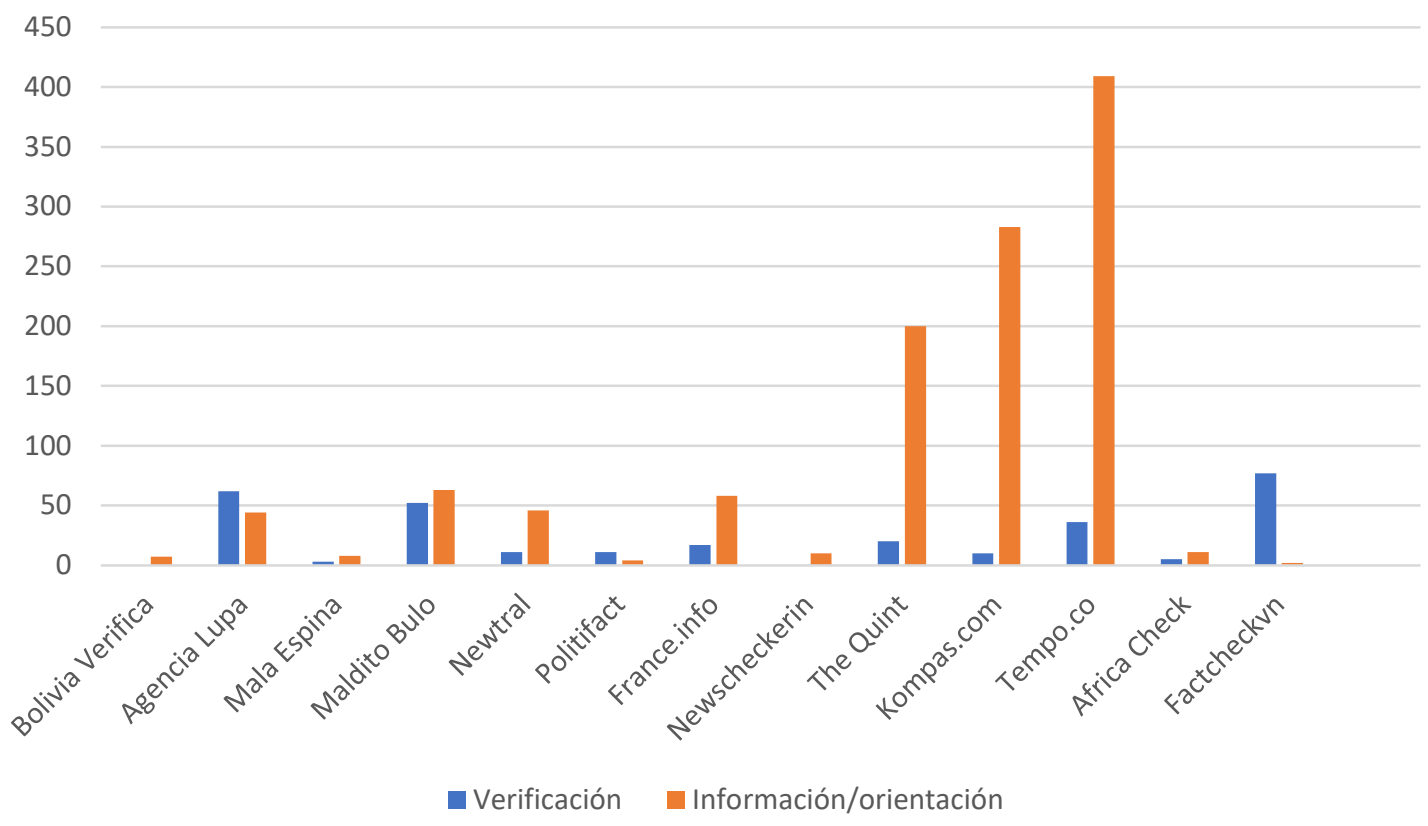

Gráfico 5: Intención de las publicaciones de los fact-checkers en TikTok desde su inicio de actividades en la red social hasta el 28 de febrero 2021

Fuente: elaboración propia

Por otra parte, en cuanto al tipo de trabajo realizado por los fact-checkers en TikTok y en referencia a la RQ2, según datos de la Tabla 2 y Gráfico 6 queda en evidencia que los contenidos adaptados a las características estéticas y narrativas de la red social constituyen el mayor porcentaje de trabajo, quedando muy por debajo aquellos que son respuestas a los comentarios de los seguidores o contenidos de otras plataformas digitales que han sido redirigidos o reciclados en TikTok. De esta manera, no se cumple la H2 y sí la H5.

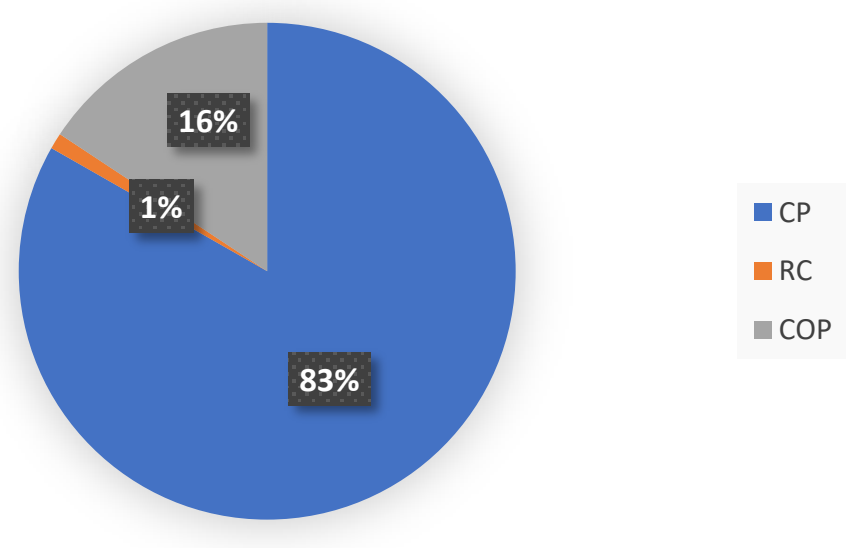

Gráfico 6: Total del tipo de contenido publicado por los fact-checkers en TikTok desde su primera publicación hasta el 28 de febrero 2021

Fuente: elaboración propia

No obstante, hay excepciones como The Quint, cuya producción multimedia en TikTok proviene de otras plataformas digitales, especialmente de Instagram. 
En lo concerniente a las encuestas, 383 encuestados se pronunciaron sobre el hecho de seguir medios o perfiles periodísticos en TikTok. De ellos, 342 (89\%) negaron hacerlo (Gráfico 7).

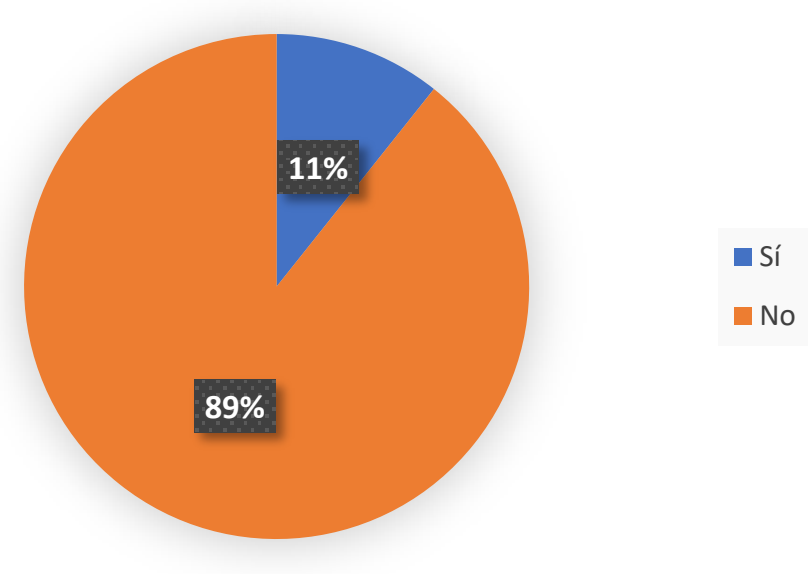

Gráfico 7: ¿Sigues a medios o perfiles periodísticos en TikTok?

Fuente: elaboración propia

Ante la pregunta específica sobre si conocían en qué consistía el fact-checking, de los 382 que respondieron apenas 170 (45\%) lo hicieron de forma afirmativa (Gráfico 8), mientras que la mayoría contestó no conocerlo.

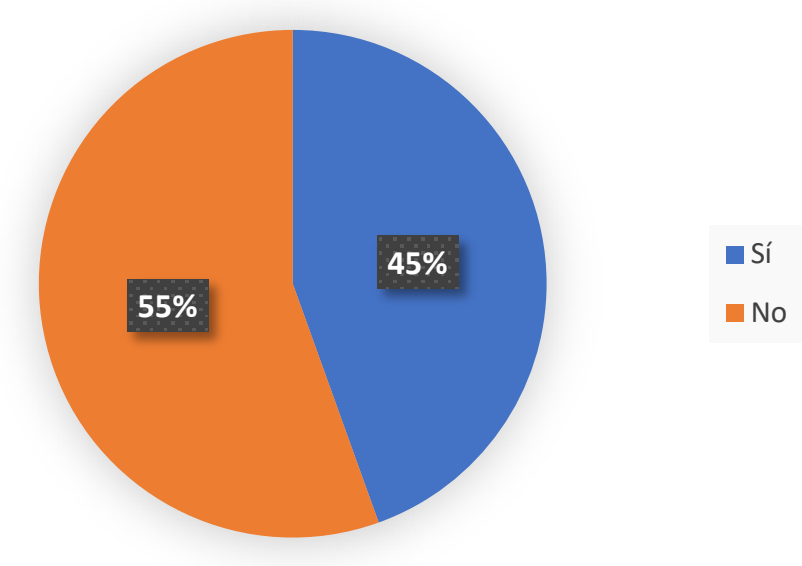

Gráfico 8: ¿Sabes en qué consiste el fact-checking?

Fuente: elaboración propia

Ante la pregunta de si seguían alguna cuenta que refiriese a procesos de fact-checking en TikTok, de 379 respuestas, 370 (98\%) fueron negativas (Gráfico 9). Entre los nombres referidos en las pocas respuestas positivas están: Maldito Bulo y Newtral en España y Agencia Lupa en Brasil. 




Gráfico 9: ¿Sigues perfiles en TikTok que refieran a procesos de fact-checking? Fuente: elaboración propia

Por último, ante la pregunta sobre hábitos de consumo de contenidos en TikTok, 218 participantes señalaron las razones por las cuales acuden a dicha red social. En este sentido, la mayor motivación es el hecho de buscar entretenimiento (41\%), seguido de conocer recetas de cocina (15\%) y descubrir nuevos destinos turísticos (11\%) (Gráfico 10).
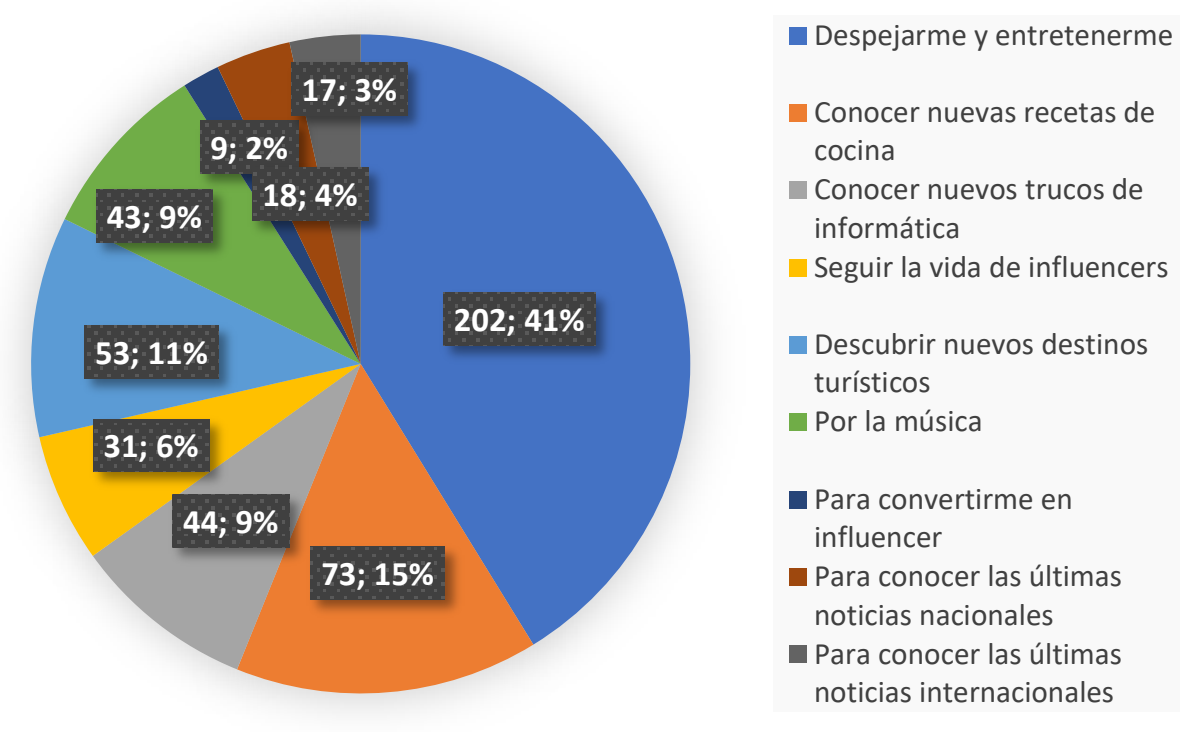

Gráfico 10: ¿Para qué utilizas TikTok comúnmente?

Fuente: elaboración propia

\section{Discusión}

Más allá de los fact-checkers con actividad en TikTok, el engagement y seguidores que reciben estos perfiles y sus contenidos, en líneas generales son bajos en comparación con algunos medios informativos de alcance global como es el caso de The Guardian (más de 3000 seguidores y más de 2000 'likes'), USA Today (casi 1 millón de seguidores y más de 23 millones de 'likes'), BuzzFeed News (más de 5000 seguidores y más de 220 mil 'likes'), Huffington Post (más de 50 mil seguidores y más de 30 mil 'likes') y NBC News (más de 600 mil seguidores y casi 20 millones de 'likes'). 
No obstante, hay excepciones como Agencia Lupa (Brasil), France.info (Francia), Kompas.com (Indonesia) y Tempo.co (Indonesia) que tienen una base importante de audiencia que reacciona a su trabajo, ayudando a la visibilidad de los contenidos de cara al algoritmo de la red social en cuestión.

Estos resultados contrastan con el caso de la agencia de verificación española Maldito Bulo que, pese a tener menos seguidores que los casos anteriores, acumula mayor engagement. Esto indica que su trabajo está resultando de interés y utilidad y, por tanto, se trata de un caso efectivo.

De esta manera, como se ha apuntado, se confirma la primera hipótesis planteada sobre el hecho de que todavía en 2021 el trabajo de estos fact-checkers es incipiente y limitado en cuanto actores en TikTok, con las excepciones señaladas.

En un primer momento, esto puede considerarse como una muestra de que la labor de verificación de medios y periodistas está enfocada a la mera experimentación. Así lo corroboran algunos de los contenidos estudiados, como los publicados por los fact-checkers Tempo.co (Indonesia) y The Quint (India). En estos se aprecia un alto volumen de contenidos correspondientes a 2020 que no están adaptados a la narrativa propuesta por TikTok, lo cual indica la premura de algunos medios de sumar esfuerzos a través de la plataforma digital tendencia del año, con la eventual poca comprensión del código del mensaje que ello implica. De hecho, algunos contenidos tienen muy poco engagement, como una entrevista a un médico (véase http://bit.ly/3qCMDG9) que corresponde a un vídeo de IGTV (véase https://bit.ly/2N2f7LS).

En concreto, los contenidos de Tempo.co son en estricto formato vertical, pero no se trata de contenidos producidos a partir de las propias herramientas de edición de TikTok, sino que han sido trabajados externamente de forma previa y carecen del dinamismo y estímulo visual que demanda la comunidad de usuarios de la red social (véase por ejemplo http://bit.ly/3vaFSzb). En otras ocasiones, editan igualmente a través de alguna herramienta externa el contenido de forma vertical, pero le insertan vídeos horizontales que, por el formato de TikTok, no encajan correctamente con la disposición de la pantalla, resultando más pequeña la propuesta multimedia y, por tanto, ajena a la estética y código de mensaje de la red social (véase http://bit.ly/3ewil5Q).

En cuanto a The Quint (India), sus publicaciones son en tono cómico y entretenido, pero muchos vídeos por sus dimensiones no parecen contenidos nativos de TikTok, sino provenientes de plataformas alternativas como pueden ser YouTube o Instagram. Este tipo de propuestas parecen ir en concordancia con los gustos y pretensiones de la audiencia a través de dicha red social, que acude a esta para despejarse y entretenerse.

Sin embargo, si nos referimos a la comprensión creativa del 'formato TikTok' en Agencia Lupa (Brasil), a partir de publicaciones tituladas 'Caiu na Rede', recogen y someten a verificación en 60 segundos información de diversa índole que circula en las comunidades populares, (véase por ejemplo http://bit.ly/3ePiXna). Este fact-checker también ha llevado a cabo la verificación de noticias a partir de una propuesta estética basada en bailes (véase http://bit.ly/2Oe0F3X).

De forma similar, Factcheckvn (Vietnam) ha desarrollado varios raps (véase http://bit.ly/3cqaZhz y http://bit.ly/3lfunS9) con el fin de innovar en este tipo de proceso, aunque llama la atención que no son los vídeos con mayor engagement y, sin duda, resultan bastantes disruptivos para la propuesta periodística en cuestión. Ambos casos son claros intentos de adaptación y desarrollo de nuevas estrategias por parte de dichos medios con respecto al código de mensaje de TikTok empleado por las audiencias más jóvenes. 
También cabe señalar nuevamente a Maldito Bulo (España). En su propuesta de verificación a veces aborda temas de cultura popular como, por ejemplo, mecanismos para "evitar llorar" cuando se corta una cebolla (véase http://bit.ly/3bo7n0b), lo cual se adapta a las propuestas que son tendencia en TikTok, en este caso en relación con el formato de los 'micro-tutoriales', un estilo narrativo propio de la red social derivado de sus propias características de vídeos de formato corto.

Por su parte, Kompas.com (Indonesia) aplica la "verificación inversa" en temas mainstream aludiendo a contenidos sobre situaciones inciertas o ejemplos contraproducentes por irresponsabilidad. Valga como muestra un vídeo (véase http://bit.ly/313PAP4) donde una mujer comenta en TikTok que se ha pegado el cabello con un pegamento determinado para darle mayor fijación. La periodista, mientras contextualiza el hecho, indica los efectos contraproducentes citando inclusive recomendaciones del fabricante del producto para evitar que otros secunden la acción. De nuevo, se trata de una narrativa que tiene que ver con las particularidades de la propia red social, puesto que en TikTok la idea de comunidad de usuarios recae muchas veces en la lógica del "challenge" imitando acciones o situaciones de otros.

Africa Check (Sudáfrica) (véase http://bit.ly/3rDb0F5) destaca por aprovechar al máximo todos los recursos nativos de edición y creación multimedia de la plataforma, explotando incluso el recurso musical, que es una de las variables del algoritmo para potenciar la visibilidad de los contenidos en el feed principal. Las periodistas que intervienen en las publicaciones de este medio establecen diálogos y coreografías definidas por la comunidad de usuarios de TikTok, por lo que su propuesta es muy orgánica y concordante con el código de mensaje establecido.

De lo dicho anteriormente, es posible afirmar que, independientemente de los temas, el país o el tipo de medio, el periodismo de verificación cuenta con interesantes e innovadoras maneras de incrementar su alcance sin necesidad de caer en prácticas sensacionalistas. Por tanto, la segunda hipótesis no queda confirmada, ya que la propia narrativa de TikTok no limita la actividad de los fact-checkers, sino que constituye otra vía para acceder a otros segmentos de audiencia.

En este sentido, Pablo Hernández de Maldito Bulo destaca que "el fenómeno de la desinformación es transversal, llega a todos los segmentos de la población y usa todas las redes sociales. Por eso es importante estar presente en todas ellas, incluida TikTok (...) La desinformación ha llegado a TikTok y el mejor lugar para combatirla es allí donde se difunda". Hernández destaca especialmente el público joven que usa esta red social, la cual puede resultar especialmente vulnerable a los bulos.

Desde Newtral sostienen que "cualquier red social que acumule un número masivo de usuarios siempre va a tener que llevar a cabo, conforme aumenta ese crecimiento, un mayor trabajo de moderación si quiere evitar la expansión de contenidos dañinos, ya no solo relativos a la desinformación". Desde el medio afirman que por esta razón se han convertido en partners de TikTok en España, con el fin de intentar atajar a tiempo las noticias falsas dentro de la plataforma.

Desde otra perspectiva, desde Mala Espina (Chile) afirman que la intención de su trabajo ha radicado en un intento de llegar a nuevas audiencias, así como ir probando distintas plataformas. En otras palabras, se trata, de momento, de un proceso de experimentación, aspecto sobre el que Karla Torres (Cotejo) afirma que es una razón importante para pensar bien la participación de los medios en la plataforma, pues quienes se encarguen de llevar el perfil y realizar un eventual monitoreo de contenidos en ella deben estar muy familiarizados con su particular estilo y código del mensaje.

En referencia la tercera hipótesis planteada en la investigación se observa que el área temática con más presencia en los verificadores estudiados en TikTok es la política, seguida de los contenidos 
relacionados con la sanidad y con temas sociales. En el caso de fact-checkers como Mala Espina (Chile), Politifact (Estados Unidos), Tempo.co (Indonesia) y Bolivia Verifica (Bolivia) abundan las publicaciones informativas y de verificación sobre temas de interés político según la estética y elementos multimedia propios de TikTok, como música y animaciones en formato vertical (véase http://bit.ly/3elf610). Asimismo, en varias publicaciones del perfil de TikTok de Tempo.co (Indonesia) la atención hacia temas políticos trasciende al plano internacional, siendo las elecciones estadounidenses de finales de 2020 uno de los temas recurrentes al respecto en el período estudiado.

La presencia de los temas sanitarios se relaciona con la pandemia de la Covid-19 en la Agencia Lupa (Brasil), Africa Check (Sudáfrica), Newscheckerin (India) y The Quint (India). Por su parte, los españoles Maldito Bulo y Newtral también han abordado temas de educación sexual, cuidado de la piel y cura de heridas, entre otros, como el caso de una respuesta a un comentario de un usuario sobre la veracidad de la orina como remedio a las picaduras de medusas (véase http://bit.ly/3ekaGr5). En este contenido concreto, la periodista de Maldito Bulo no solo hace alusión al mainstream de entretenimiento televisivo, sino que referencia fuentes científicas sobre las acciones más acordes a tomar en cuenta ante dicha situación.

No obstante, en proporción general, la categoría "otros temas" es la que más abunda en la producción de contenidos de estos emisores a través de TikTok. Al respecto, es posible apreciar verificaciones o contenidos informativos/orientativos sobre temas climáticos, tecnológicos, de farándula local e internacional o cualquier otro tema que no entrara en las categorías preestablecidas para este estudio. Es decir, en la mayoría de los fact-checkers estudiados, pese a poner mucho énfasis en la verificación o desmentir temas políticos, sanitarios, económicos o sociales, resulta frecuente observar tipologías más diversas de contenidos.

De esta manera, según las evidencias aquí expuestas y tomando en consideración las presunciones de la tercera hipótesis, como se apuntaba en líneas anteriores, se puede afirmar que, en gran medida, en los fact-checkers sometidos a estudio, tanto la coyuntura de la Covid-19 como los episodios políticos a escala global coparon las publicaciones, pues se trata de temas que han sido muy susceptibles de alimentar un flujo enorme de información en muchos casos cuestionable o especulativa (López, 2020; Sidorenko, Herranz y Cantero, 2020; Pappapico, 2020).

Pese a la idea implícita que puede arrojar el anglicismo 'fact-checkers', estos medios periodísticos de verificación de información, lejos de tener una actividad exclusivamente centrada en contrastar noticias y rumores, también publican como medios generalistas y educadores en materia periodística.

Si bien la verificación está presente en la mayoría los perfiles observados, solo en la producción multimedia de Agencia Lupa (Brasil) y Factcheckvn (Vietnam) este proceso periodístico supera a la creación de contenidos informativos/orientativos. Asimismo, queda en evidencia que Bolivia Verifica (Bolivia) y Newscheckerin (India) solo se han centrado en producir con base en esta segunda premisa, que persigue de igual modo mitigar la desinformación planteando por adelantado información específica sobre diversos eventos o situaciones y así reducir la posibilidad de que surjan bulos o especulaciones al respecto.

En el caso particular de The Quint (India), su enfoque se ha orientado en buena parte a promocionar reiteradamente sus rutinas de trabajo y estrategias de verificación en tono entretenido, como ya se ha indicado. Por su parte, Maldito Bulo (España) incide mucho en la educación y orientación sobre el trato con la desinformación y las noticias falsas, o elaborando contenidos donde aconsejan cómo actuar, por ejemplo, con un familiar que acostumbra a publicar a través de aplicaciones de mensajería contenidos que no están contrastados (véase http://bit.ly/3v78U2D). 
Por tanto, no se cumple en la mayoría de los casos estudiados la cuarta hipótesis, puesto que el trabajo de los fact-checkers a través de TikTok no se ha centrado exclusivamente en verificar y desmentir bulos y noticias falsas. De hecho, las dos excepciones anteriormente señaladas pueden suponer eventualmente cierta confusión entre usuarios que aún desconocen en qué consiste el proceso de verificación de noticias o la finalidad de un fact-checker.

En este sentido, se hace referencia a un ejemplo en particular de Maldito Bulo, donde el medio contestó justamente a esta última duda (véase http://bit.ly/3chRGqK). Al respecto, Pablo Hernández (Maldito Bulo), incide en que "aparte de reaccionar a las desinformaciones para retirarlas, hay que actuar creando desmentidos en formato TikTok pero también dando información veraz a través de la misma red social".

En relación con la quinta y última hipótesis de la investigación, se evidencia que los fact-checkers interactúan poco con su audiencia a pesar de la importancia de la comunidad de usuarios en la dinámica de TikTok. Este trabajo de creación de una comunidad resulta relevante para aumentar el alcance y la relevancia de los contenidos verificados y que se aproxime al de las propias informaciones falsas, convirtiéndose así en una alternativa para dinamizar la labor educativa, orientadora y verificadora, no solo en TikTok, sino también en otras redes sociales como Twitter (Magallón-Rosa, 2018) y Facebook (Andersen y Søe, 2020). Esto invita a la reflexión dada la importancia de la comunidad de usuarios en Tiktok.

No obstante, en atención a casos particulares, entre los perfiles estudiados, Maldito Bulo (España) y The Quint (India) son los que más interacciones reportan con sus usuarios, estrechando así la relación entre medio y audiencia. Como nota adicional, Maldito Bulo ha explorado esta interacción a partir del recurso del 'duet' (dueto) ${ }^{1}$ para reaccionar a otro vídeo dentro de la misma red social (véase http://bit.ly/3kVspGt).

Desde Agencia Lupa (Brasil) afirman que la mayoría de los contenidos que verifican y sobre los cuales realizan publicaciones en TikTok, así como a través de otras redes sociales, proviene en gran medida de la aportación de los usuarios de internet, lo que plantea otro tipo de interacciones, en este caso de la audiencia con el medio.

Podría pensarse que, al haber pocos fact-checkers aún en TikTok, los que están activos podrían convertirse en referentes del periodismo de verificación. Sin embargo, los datos de las encuestas evidencian aún el desconocimiento que existe sobre la labor de estos actores o su simple presencia en la red social, pues aún no se relaciona con el entorno propicio para acudir a este tipo de contenidos y mensajes.

\section{Conclusiones}

A través de esta investigación se ha podido profundizar en las formas narrativas y en la comunicación que llevan a cabo agencias de verificación de diferentes lugares del mundo en la red social TikTok y constatar el elevado potencial de la red social para combatir los contenidos desinformativos. De hecho, debido a la proliferación de vídeos de contenido engañoso durante la pandemia mundial de la Covid-19, diversas instituciones sanitarias y humanitarias como la OMS, Cruz Roja o Unicef emplearon esta red social para desmentir contenidos falsos e informar de

\footnotetext{
${ }^{1}$ TikTok permite a partir de los duetos que la pantalla se divida en dos, teniendo en el lado izquierdo el perfil que reacciona a un contenido y en el derecho el vídeo original sobre el cual se desea comentar.
} 
medidas de prevención contra el coronavirus (Ballesteros, 2020; Fingas, 2020; Sidorenko, Herranz y Cantero, 2020), así como también lo hicieron usuarios particulares, en su mayoría, profesionales sanitarios.

Más allá del ámbito humanitario y sanitario, las agencias de verificación de los diferentes países publican periódicamente en TikTok sobre diferentes cuestiones como la política, la economía y temas sociales. De hecho, fact-checkers como Agencia Lupa (Brasil), Newtral (España) y Cotejo (Venezuela) consideran que TikTok puede ser eventualmente una plataforma digital con un potencial importante para la circulación de noticias falsas y especulaciones que obliga a medios especializados en verificación, medios informativos generalistas y periodistas a centrar su atención en estos contenidos falsos y actuar para combatir la desinformación.

Esta actividad de verificación de información y desmentidos en TikTok por parte de los factcheckers se adapta a la narrativa propia de la red social en la mayoría de los casos estudiados, empleando el vídeo vertical, herramientas de edición propias de TikTok y formatos como los microtutoriales. Esto se puede atribuir a la competencia por captar la atención de los usuarios en la red social con contenidos más entretenidos y visuales. Si los usuarios los ignoran, el algoritmo de la red social opta por no seguir mostrándolos en el feed principal.

Asimismo, se observa una falta de interacción entre los perfiles de las plataformas de verificación y la audiencia, lo cual resulta fundamental para el desarrollo del fact-checking (Elizabeth y Mantzarlis, 2016). La lógica de la comunicación a través de TikTok se basa en un constante llamado a la acción, bien a través de engagement, bien emulando acciones y contenidos por parte de terceros dada la importancia de la comunidad de usuarios en TikTok. Este debe ser un elemento sobre el cual muchos emisores deben incidir más.

Una mayor comunicación con la audiencia podría contribuir a aumentar el engagement de los perfiles de las agencias de verificación en TikTok, así como una presencia más regular en un momento en que el periodismo de verificación de datos en países como España todavía se encuentra en una fase incipiente (López y Rodríguez, 2020).

Así, el trabajo de fact-checkers, medios y periodistas en TikTok es crear contenidos atractivos, dinámicos y estimulantes visualmente que compitan con la basta producción de creatividad que abunda en la plataforma, sin desmerecer, resentir ni desmejorar la exposición periodística realizada. En otras palabras, el periodismo de verificación tiene cabida y perdurará en TikTok siempre y cuando procure y desarrolle nuevas aptitudes narrativas, tomando en consideración la constante evolución que experimenta la plataforma en cuanto a tendencias y grupos de usuarios.

Por otra parte, la presencia de las agencias de verificación en TikTok resulta fundamental para llegar a un mayor número de usuarios, sobre todo a los jóvenes, un segmento de la población especialmente vulnerable a los bulos, como se ha señalado en la investigación. Por tanto, sería deseable una mayor expansión en esta red de social de estos contenidos tan necesarios en la sociedad de la posverdad, pero se debe tener en cuenta que no es habitual en los usuarios de TikTok dirigirse expresamente a un perfil en especial para consumir su contenido, pues los estímulos visuales de la plataforma se centran -y obligan a los usuarios a centrar su atención- justamente en el feed principal.

A su vez, se debe trabajar en una alfabetización mediática y en la responsabilidad de la ciudadanía (Aguaded y Romero, 2015; Pabón-Montealegre, 2020) para controlar la difusión de informaciones falsas, además de acudir a fuentes fiables para informarse (Guallar et al, 2020; Palomo y Sedano 2018). Los medios y periodistas deben mantener e incrementar los esfuerzos educativos sobre el 
peligro que representa la desinformación y la importancia de diversos mecanismos para contrarrestarla.

Asimismo, la propia narrativa y códigos de TikTok, así como su audiencia y elementos de engagement, la diferencian de otras redes sociales como Facebook y Twitter, lo que ha supuesto un límite en la investigación para establecer una posible comparativa con la presencia y actividad de fact-checkers en estas redes sociales. Resultaría interesante realizar una comparativa en futuras investigaciones delimitando un período concreto o la verificación de un tema determinado por parte de los fact-checkers en las diferentes redes sociales.

En definitiva, se considera que TikTok tiene un gran potencial para el periodismo de verificación trabajando desde la propia narrativa de la red social y fomentando la interacción con la audiencia. En este sentido, también sería interesante centrar futuras investigaciones en el análisis de contenido de estas interacciones y del engagement en los perfiles de verificación presentes en TikTok.

\section{Bibliografía}

Aguaded, I. y Romero R., L. M. (2015). Mediamorfosis y desinformación en la infoesfera: Alfabetización mediática, digital e informacional ante los cambios de hábitos de consumo informativo. Education in the Knowledge Society, 16(1), 44-57.

Albright, J. (2017). Welcome to the Era of Fake News. Media and Communication, 5 (2), 87-89. http://dx.doi.org/10.17645/mac.v5i2.977

Allcott, H., Gentzknow, M. y Yu, C. (2019). Trends in the diffusion of misinformation on social media. Research \& Politics, (6), 2, 1-8. https://doi.org/10.1177/2053168019848554

Allcott, H. y Gentzkow, M. (2017). Social media and fake news in the 2016 election. Journal of Economic Perspectives, 31(2), 211-236.

Alonso G., Marián (2019). Fake News: desinformación en la era de la sociedad de la información. En:Ámbitos. Revista internacional de Comunicación, $45 . \quad 29-52$. http://dx.doi.org/10.12795/Ambitos.2019.i45.03

Amorós G., M. (2018). Fake News. La verdad de las noticias falsas. Plataforma Editorial.

Andersen, J. y \& Søe, S. O. (2020). Communicative actions we live by: The problem with factchecking, tagging or flagging fake news - the case of Facebook. European Journal of Communication, 35(2), 126-139. https://doi.org/10.1177/0267323119894489

Ballesteros H., C.A. (2020). La propagación digital del coronavirus: Midiendo el engagement del entretenimiento en la red social emergente TikTok. Revista Española de Comunicación en Salud. Suplemento 1, 171-185. https://doi.org/10.20318/recs.2020.5459

Basch, C. H., Hillyer, G. C. y Jaimie, C. (2020). COVID-19 on TikTok: harnessing an emerging social media platform to convey important public health messages. International Journal of Adolescent Medicine and Health, 1-3. https://doi.org/10.1515/ijamh-2020-0111 
Blanco A., I., García G., C., y Tejedor C., S. (2019). El impacto de las fake news en la investigación en Ciencias Sociales. Revisión bibliográfica sistematizada. Historia Y Comunicación Social, 24(2), 449-469. https://doi.org/10.5209/hics.66290

Burkhardt, J. M. (2017). Chapter 1. History of Fake News. Library Technology Reports, 53(8), 5-9.

Cabezuelo L., F. y Manfredi, J. L. (2019). Posverdad, fake-news y agenda política en el discurso de Trump en Twitter. Historia y comunicación social, 24 (2), 449-483.

Ceron, W., De-Lima-Santos, M.F. y Quiles, M. G. (2021), Fake news agenda in the era of COVID19: Identifying trends through fact-checking content, Online Social Networks and Media, 21, https://doi.org/10.1016/j.osnem.2020.100116

Coleman, S., Morrison, D. E. y Anthony, S. (2012). A Constructivist Study of Trust in the News. Journalism Studies, 13, (1). 37-53. doi: 10.1080/1461670X.2011.592353

Coughlan, S. (2017, 12 de enero). What does post-truth mean for a philosopher? BBC News. Consultado el 11 de marzo 2021 en: https://www.bbc.com/news/education-38557838

Del Fresno G., M. (2019). Desórdenes informativos: sobreexpuestos e infrainformados en la era de la posverdad. Profesional de la información, $28 \quad$ (3), e280302. https://doi.org/10.3145/epi.2019.may.02

Ditrendia (2020). Móviles en España y en el Mundo 2020. Consultado el 15 de febrero 2021 en: https://bit.ly/3iY6iwV

Egelhofer, J.N., Aaldering, L., Eberl, J.M., Galyga S. y Lecheler, S. (2020) From Novelty to Normalization? How Journalists Use the Term "Fake News" in their Reporting. Journalism Studies, 21(10), 1323-1343. https://doi.org/10.1080/1461670X.2020.1745667

Elizabeth, J. y Mantzarlis. A. (2016, 6 de julio). The fact is, fact-checking can be better. American Press Institute. Consultado el 10 de marzo $2021 \mathrm{en}$ : https://www.americanpressinstitute.org/factchecking-project/fact-not-fact-checking-great/

European Commission (2018). A multi-dimensional approach to disinformation. Report of the independent High-Level Group on Fake News and Online Disinformation. Directorate-General for Communication Networks, Content and Technology. Unión Europea.

Fingas, J. (2020, 3 de marzo). La OMS se une a TikTok para combatir la desinformación del coronavirus. Entrepeneur. Consultado el 15 de marzo 2021 en: https://www.entrepreneur.com/article/347084

Fundacion Gabo. (2020, Abril, 2). Más de 100 sitios para verificar desinformación sobre Covid-19. Consultado el 14 de junio 2021 en: https://fundaciongabo.org/es/noticias/mas-de-100-sitios-paraverificar-desinformacion-sobre-el-covid-19

Gaitán, J. A. y Piñuel, J. L. (1998). Técnicas de investigación en Comunicación Social. Síntesis. 
Guallar, J., Codina, Ll., Freixa, P. y Pérez M., M. (2020). Desinformación, bulos, curación y verificación. Revisión de estudios en Iberoamérica 2017-2020. Telos: revista de Estudios Interdisciplinarios en Ciencias Sociales, 22 (3), 595-613. https://doi.org/10.36390/telos223.09

IFCN. (n.d). Verified signatories of the IFCN code of principles. Consultado el 14 de junio 2021 en: https://ifcncodeofprinciples.poynter.org/signatories

Jang, S. y Kim, J.K. (2018). Third person effects of fakenews: Fakenews regulation and media literacy interventions. Computers in human Behavior, 80, 295-302.

Keyes, R. (2004). The Post-truth Era: Dishonesty and deception in contemporary life. St. Martins Pr.

Lazer, D. M. J., Baum, M. A., Benkler, Y., Berinsky, A. J., Greenhill, K. M., Menczer, F., Metzger, Miriam J., Nyhan, B., Pennycook, G., Rothschild, D., Schudson, M., Sloman, Steven A., Sunstein, C. R., Thorson, E. A., Watts, Duncan J., Zittrain, J. L. (2018). The science of fake news. Science, v. 359, n. 6380, 1094-1096. https://doi.org/10.1126/science.aao2998

Li, X., Xiaohui, Y. y Zhengwu, Z. (2019). Research on the causes of the "TikTok" app becoming popular and the existing problems. Journal of advanced management science, 7(2), 59-63. https://doi.org/10.18178/joams.7.2.59-63

López, E. (2020). Las pandemias en el siglo XXI: Un desafío para la salud pública. Actualización en Nutrición, 21 (1), 1-3

López-Borrull, A. (2020). Fake news e infodemia científica durante la Covid-19, ¿dos caras de la misma crisis informacional?. Anuario ThinkEPI, 14, e14e07

López P., F., y Rodríguez R., J. (2020). El Fact-checking en España. Plataformas, prácticas y rasgos distintivos. Estudios Sobre El Mensaje Periodístico, 26(3), 1045-1065. https://doi.org/10.5209/esmp.65246

Magallón-Rosa, R. (2018). Nuevos formatos de verificación. El caso de Maldito Bulo en Twitter. Sphera Publica, 1 (18), 41-65.

MacKenzie, A. y Bhatt, I. (2020) Lies, Bullshit and Fake News. Postdigit Sci Educ 2. https://doi.org/10.1007/s42438-019-00085-4

McNair, B. (2017). Fake News: Falsehood, Fabrication and Fantasy in Journalism. Routledge.

Mazaira C, A., Rúas A., J. y Puentes R., I. (2019). Fact-checking en los debates electorales televisados de las elecciones generales de 2015 y 2016. Revista Latina de Comunicación Social, 74, 748 a 766. http://dx.doi.org/10.4185/RLCS-2019-1355

Nyhan, B. y Reifler, J. (2015), The Effect of Fact-Checking on Elites: A Field Experiment on U.S. State Legislators. American Journal of Political Science, 59, 628640. https://doi.org/10.1111/ajps.12162

Omar, B. y Dequan, W. (2020). Watch, Share or Create: The Influence of Personality Traits and User Motivation on TikTok Mobile Video Usage. International Journal of Interactive Mobile Technologies, 14 (4), 121-137. https://doi.org/10.3991/ijim.v14i04.12429 
Pabón-Montealegre, M. V. (2020). Criterio para navegar en la desinformación. La WebQuest como herramienta de aprendizaje para formar a los maestros. Universitas Científica, 16(1), 18-21.

Palau-Sampio, D. (2018). Fact-checking y vigilancia del poder: La verificación del discurso público en los nuevos medios de américa Latina. Comunicación y Sociedad, 31 (3), 347-365.

Palomo, B., y Sedano A., J. A. (2018). WhatsApp como herramienta de verificación de fake news. El caso de B de Bulo. Revista Latina, (73), 1384-1397. https://doi.org/10.4185/RLCS-2018-1312

Papapicco, C. (2020). Informative contagion: The Coronavirus (COVID-19) in italian journalism. Online Journal of Communication and Media Technologies, 10 (3), 1- 12

Pérez, S. (2021, 3 de febrero). TikTok to flag and downmark 'unsubstantiated' claims fact checkers can’t verify. TechCruch. Consultado el 11 de marzo 2021 en: https://tcrn.ch/3w3G5nY

Pérez-Curiel, C., y Velasco-Molpeceres, A. M. (2020). Impacto del discurso político en la difusión de bulos sobre Covid-19. Influencia de la desinformación en públicos y medios. Revista Latina, (78), 86-119. https://doi.org/10.4185/RLCS-2020-1469

Pérez-Dasilva, J.A., Meso-Ayerdi, K. y Mendiguren-Galdospín, T. (2020). Fake news y coronavirus: detección de los principales actores y tendencias a través del análisis de las conversaciones en Twitter. Profesional de la información, 29 (3). https://doi.org/10.3145/epi.2020.may.08

Preston, S., Anderson, A., Robertson, D.J., Shephard, M.P. y Huhe, N. (2021). Detecting fake news on Facebook: The role of emotional intelligence. PLoS ONE, 16(3). https://doi.org/10.1371/journal.pone.0246757

Rapkin, M. (2017, 1 de noviembre). The social media platform that has Gen Z obsessed. Wall Street Journal Magazine. Consultado el 15 de marzo 2021 en: https://on.wsj.com/2YVWhZj

Rodríguez-Fernández, L. (2021). Propaganda digital. Comunicación en tiempos de desinformación. Barcelona: UOC.

Salas A., C. (2019). La primera fake news de la historia. Historia y comunicación social, 24 (2), 411 431. https://dx.doi.org/10.5209/hics.66268

Salaverría, R., Buslón, N., López P., F., León, B., López G., I., Erviti, M. C. (2020). Desinformación en tiempos de pandemia: tipología de los bulos sobre la Covid-19. Profesional de la información, 29 (3). https://doi.org/10.3145/epi.2020.may.15

Salaverría, R. (2020). Claves para hacer frente a la desinformación. Biko INSIGHTS. 52-61.

Sánchez D., J.M. y Magallón R., R. (2020). Infodemia y COVID-19. Evolución y viralización de informaciones falsas en España. Revista Española de Comunicación en Salud. Suplemento 1. 31 41. https://doi.org/10.20318/recs.2020.541

Shuai, Y., Yuzhen, Z. y Yifang, M. (2019). Analysis of the reasons and development of short video application - Taking Tik Tok as an example. 9th International Conference on Information and 
Social Science (ICISS 2019). Francis Academic Press, 340-343. https://doi.org/10.25236/iciss.2019.062

Sidorenko B., P., Herranz, J. M. y Cantero, J. I. (2020). Use of new narratives for COVID-19 reporting: from $360^{\circ}$ videos to ephemeral TikTok videos in online media. Tripodos, 47(1), 105122

Soler, P., Enríquez, A. M. (2012). Reflexión sobre el rigor científico en la investigación cualitativa. Estudios sobre el Mensaje Periodístico, 18, 879-888. https://doi.org/ggvt53

Terol B., R. y Alonso- L., N. (2020). La prensa española en la Era de la Posverdad: el compromiso de la verificación de datos para combatir las Fake News. Revista Prisma Social, (31), 304-327

Tesich, S. (1992). A goverment of lies. The Nation, 254(1)

Torres M., J. L. y Villena A., E. (2020) Usos y recursos de TikTok como herramienta de comunicación. En, Herrero G., J., Segarra S., J. e Hidalgo M., T. (coords.) La narrativa audiovisual. Del concepto a la alfabetización mediática (259-269) Madrid: Fragua.

Ufarte, M.J., Peralta, L., Murcia, F.J. (2018). Fact-checking: un nuevo desafío del periodismo. Profesional de la Información, 27(4), 733-741. https://doi.org/10.3145/epi.2018.jul.02

Vásquez H., J., Negreira R., M. C. y López G., X. (2020). Let's dance the news! How the news media are adapting to the logic of TikTok. Journalism, 1-19. https://doi.org/10.1177\%2F1464884920969092

Vázquez H., J., Vizoso, Á. y López G., X. (2019). Innovación tecnológica y comunicativa para combatir la desinformación: 135 experiencias para un cambio de rumbo. El profesional de la información, 28(3). https://doi.org/10.3145/epi.2019.may.01

Van Den Bremer, A. J, M. y Siebelink, R. (2020). Critical Thinking on TikTok. Tesis sin publicar. Universidad de Utrecht.

Vizoso, A. y Vázquez H., J. (2019). Plataformas de fact-checking en español. Características, organización y método. Communication \& Society, 32(1), 127-144. https://doi.org/10.15581/003.32.1.127-144

Vogel, E. A. y Rose, J. P. (2016). Self-reflection and interpersonal connection: Making the most of self-presentation on social media. Translational Issues in Psychological Science, 2, 294-302. https://doi.org/10.1037/tps0000076

Yu-Liang, F. Y. L., Chun-Chin, C. y Shu-Ming, W. (2019). Evaluation of charm factors of short video user experience using FAHP - a case study of TikTok app. IOP Conference Series: Materials Science and Engineering, 688, 1-5. https://doi.org/10.1088/1757-899X/688/5/055068 


\section{AUTORES}

\section{Pavel Sidorenko Bautista}

Doctor por la Facultad de Comunicación de la Universidad de Castilla-La Mancha. Profesor en la Facultad de Ciencias de la Comunicación de la Universidad Francisco de Vitoria y del Máster en Comunicación e Identidad Corporativa de la Universidad Internacional de La Rioja. Investiga sobre nuevas narrativas y nuevas tecnologías aplicadas a la comunicación y el periodismo. Pertenece al grupo de investigación 'DiploCity'.

pavel.sidorenko@ufv.es

\section{Índice H: 5}

Orcid ID: https://orcid.org/0000-0002-8094-3089

Google Scholar: https://scholar.google.es/citations?hl=es\&user=NZkQkeEAAAAJ

ResearchGate: https://www.researchgate.net/profile/Pavel-Sidorenko-Bautista

Scopus ID: https://www.scopus.com/authid/detail.uri?authorId=57211801795

Academia.edu: https://ufvitoria.academia.edu/PavelSidorenkoBautista

\section{Nadia Alonso López}

Doctora en Comunicación por la UV y Máster en Gestión de la Producción Audiovisual y en Marketing y Comunicación Digital. Profesora en el Departamento de Comunicación Audiovisual, Documentación e Historia del Arte de la Universitat Politècnica de València. Pertenece al grupo de investigación ArtiCom y es miembro de la Asociación Española de Investigación de la Comunicación (AE-IC) y de la Sociedad Española de Periodística (SEP).

naallo1@ har.upv.es

\section{Índice H: 3}

Orcid ID: http://orcid.org/0000-0002-5220-2232

Google Scholar: $\underline{\text { https://scholar.google.es/citations?user=77kOgs8AAAAJ\&hl=es }}$

ResearchGate: https://www.researchgate.net/profile/Nadia-Alonso-Lopez

Academia.edu: https://upv-es.academia.edu/NadiaAlonsoL\%C3\%B3pez

\section{Fábio Giacomelli}

Periodista. Estudiante de doctorado en Ciencias de la Comunicación e Investigador asociado al LabCom.CA en la Universidad Beira Interior. Financiado por una beca doctoral FCT con referencia SFRH/BD/143944/2019

fabio@ubi.pt

Índice H: 1

Orcid ID: https://orcid.org/0000-0003-3261-0041

Google Scholar: https://scholar.google.com/citations?user=ZRC7_6wAAAAJ\&hl=pt-BR\&oi=ao

ResearchGate: https://www.researchgate.net/profile/Fabio-Giacomelli-3

Academia.edu: http://www.ubi.academia.edu/fabiogiacomelli 